\title{
Dipeptidyl aminopeptidase processing and biosynthesis of alkaline extracellular protease from Yarrowia lipolytica
}

\author{
Sam Matoba, ${ }^{1}$ Kevin A. Morano, ${ }^{2} \dagger$ Daniel J. Klionsky, ${ }^{2}$ Keunsung Kim ${ }^{1}$ \\ and David M. Ogrydziak'
}

Institute of Marine Resources ${ }^{1}$ and Section of Microbiology², University of California, Davis, CA 95616, USA
Author for correspondence: David M. Ogrydziak. Tel: +1 916752 2506. Fax: +1 9167524759. e-mail:dmogrydziak@ucdavis.edu

Keywords: Yarrowia lipolytica, protease, dipeptidyl aminopeptidase

\section{INTRODUCTION}

The dimorphic yeast Yarrowia lipolytica secretes high levels of a $32 \mathrm{kDa}$ subtilisin-like alkaline extracellular protease (AEP) (Tobe et al., 1976; Ogrydziak \& Scharf, 1982; Matoba et al., 1988). AEP has been used as a reporter molecule for in vivo studies of functions of the signal recognition particle of Y. lipolytica ( $\mathrm{He}$ et al., 1992; Yaver et al., 1992), and the XPR2 gene (encoding AEP) promoter and prepro-region have been used for heterologous protein secretion. AEP is synthesized with a 157 aa prepro-region that begins with a signal sequence

†Present address: Department of Biological Chemistry, University of Michigan Medical School, Ann Arbor, MI 48109-0606, USA.

Abbreviations: AEP, alkaline extracellular protease; DPAPase, dipeptidyl aminopeptidase; FOA, fluoroorotic acid. followed by a stretch of nine X-Ala, X-Pro dipeptides (Fig. 1). The prepro-region contains a consensus sequence for $\mathrm{N}$-linked glycosylation and ends with a LysArg pair of basic amino acids (Davidow et al., 1987; Matoba et al., 1988; Nicaud et al., 1989). Removal of the signal peptide and addition of $2 \mathrm{kDa}$ of $N$-linked carbohydrate in the pro-region are thought to occur as prepro-AEP is translocated across the ER membrane yielding a $55 \mathrm{kDa}$ AEP precursor (Matoba et al., 1988; Fabre et al., 1991). Removal of the X-Ala, X-Pro dipeptides by dipeptidyl aminopeptidase (DPAPase) activity, which yields a $52 \mathrm{kDa}$ precursor, and cleavage after Lys-Arg at the end of the pro-region by the KEX2like XPR 6 processing endoprotease, which yields $32 \mathrm{kDa}$ mature AEP, presumably occur in the late Golgi (Matoba et al., 1988; Matoba \& Ogrydziak, 1989; Fabre et al., 1991; Enderlin \& Ogrydziak, 1993; Lopez et al., 1994). 
(a)

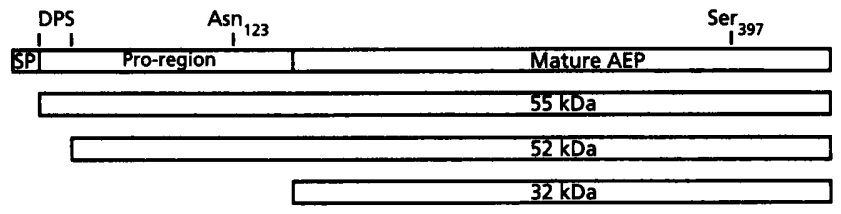

(b)

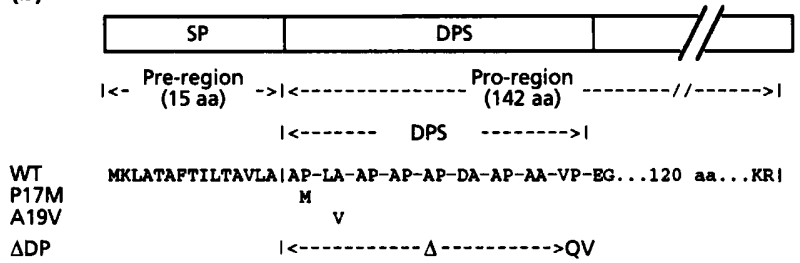

Fig. 1. AEP processing. SP, signal peptide; DPS, dipeptide stretch. (a) Entire AEP protein (454 aa), 55 and $52 \mathrm{kDa}$ AEP precursors and mature AEP. (b) The prepro-region of AEP and mutations of the dipeptide stretch. Vertical lines in the wildtype (WT) (CX161-1B) sequence indicate positions of signal peptidase and Xpr6p endoprotease processing.

The role of the nine X-Ala, X-Pro dipeptides (dipeptide stretch) in AEP processing and secretion is not clear. The location of the dipeptide stretch in AEP (over 100 aa upstream of the $\mathrm{N}$ terminus of mature AEP) is unusual in that in other cases, such as honey bee prepromellitin and prepro- $\alpha$-factor, the dipeptides are located directly upstream of the mature polypeptide and their removal results in activation of the polypeptide (Kreil, 1990). The lack of activity of the $52 \mathrm{kDa}$ AEP precursor secreted by $x p r 6$ mutants indicates that removal of the dipeptide stretch does not result in AEP activation (Matoba \& Ogrydziak, 1989). We speculated that XPR6 processing, which does result in AEP activation, was inhibited until the dipeptide stretch was removed and that this provided a mechanism for preventing premature activation of AEP in the secretory pathway (Matoba \& Ogrydziak, 1989).

The pro-region seems to serve several functions. Its removal is necessary for enzyme activation. The prodomain may also function as an internal chaperone to promote AEP folding to secretion-compatible structures and as a template for the final folding involved in AEP activation. Three different deletions of the AEP proregion (removal from the end of the dipeptide stretch to $\mathrm{Asp}_{128}$, removal of all the pro-region after the dipeptide stretch and removal of the region between Lys-Arg ${ }_{60}$ and the end of the pro-region) resulted in intracellular accumulation of AEP precursors of the predicted size but no AEP activity or AEP-related proteins were secreted (Fabre et al., 1991). These AEP precursors were translocated into the ER but did not undergo DPAPase or Xpr6p processing, suggesting they had not reached the late Golgi (Fabre et al., 1991). Supplying the AEP prepro-region in trans resulted in significant but slower secretion of partially active AEP from the deletion constructs (Fabre et al., 1992).
$\mathrm{N}$-Linked glycosylation in the pro-region is needed for AEP secretion at higher temperatures (Fabre et al., 1991). After elimination of the $\mathrm{N}$-linked glycosylation site at $\mathrm{Asn}_{123}$, AEP was secreted at $18{ }^{\circ} \mathrm{C}$ but not at $28^{\circ} \mathrm{C}$. A $53 \mathrm{kDa}$ intracellular AEP precursor was detected at $28^{\circ} \mathrm{C}$ but not at $18^{\circ} \mathrm{C}$, suggesting that misfolding was occurring at the higher temperature and that the misfolded protein was retained in the secretory pathway somewhere before the late Golgi.

Because of the high levels of secretion of AEP, the XPR2 promoter and parts of the prepro-region have been used to secrete foreign proteins in Y. lipolytica, including prochymosin (Franke et al., 1988), Saccharomyces cerevisiae invertase (Nicaud et al., 1989), porcine $\alpha 1-$ interferon (Heslot et al., 1989), hepatitis B virus middle surface antigen (Hamsa \& Chattoo, 1994) and bovine $\beta$ lactoglobulin (M. Huang \& D. M. Ogrydziak, unpublished results). Interestingly, of six XPR2-human blood coagulation factor XIIIa fusion constructs containing increasing portions of the AEP prepro-region, only the one containing the signal peptide and dipeptide stretch secreted human blood coagulation factor XIIIa (Tharaud et al., 1992). Improved understanding of the role of the dipeptide stretch will facilitate production of commercially desirable heterologous proteins by Y. lipolytica.

In this study we have examined several aspects of AEP processing and secretion. We determined the site of signal peptide cleavage and that AEP does not undergo autocatalytic processing during biosynthesis. We were particularly interested in determining the role of the dipeptide stretch and DPAPase processing in AEP maturation. These were investigated by altering the dipeptide stretch, using Pro-boroPro to inhibit DPAPase processing, by deleting the dipeptide stretch and by expressing AEP in S. cerevisiae strains with and without DPAPase activity. The two major processing events carried out by DPAPase and XPR6 seem to occur independently.

\section{METHODS}

Materials. The Random Primed DNA Labelling Kit and proteinase $\mathrm{K}$ were purchased from Boehringer Mannheim. L- $\left[4,5-{ }^{3} \mathrm{H}\right]$ Leu $\left(4.44 \times 10^{12}-7.03 \times 10^{12} \mathrm{~Bq} \mathrm{mmol}^{-1}\right)$ and L$\left[{ }^{35} \mathrm{~S}\right]$ Met $\left(>2.96 \times 10^{13} \mathrm{~Bq} \mathrm{mmol}^{-1}\right)$ were purchased from Amersham. T4 DNA polymerase and T4 polynucleotide kinase were from New England Biolabs. Restriction enzymes and T4 DNA ligase were purchased from all the above suppliers and from BRL and Stratagene. Oligonucleotides were purchased from Operon Technologies Inc. Galactose and Triton X-100 were from Sigma and bovine serum albumin, fraction V from USB. PMSF, casein (Hammersten), polypropylene glycol, DMSO, Triton X-100, EDTA, Protein ASepharose 4 Fast Flow, endoglycosidase $\mathrm{H}$ and 5 -fluoroorotic acid (FOA) were obtained from the same sources as described previously (Yaver et al., 1992). AEP antiserum was prepared as described previously (Matoba et al., 1988).

Media. LB, 2XYT, H medium and glucose-minimal medium were used for growing Escherichia coli and in vitro mutagenesis (Sambrook et al., 1989; Bio-Rad Muta-Gene M13 In vitro 
Table 1. Yeast strains used in this study

\begin{tabular}{|c|c|c|}
\hline Strain & Genotype & Source \\
\hline \multicolumn{3}{|l|}{ S. cerevisiae } \\
\hline INV Sc1 & $\alpha$ bis $3-\Delta 1$ leu 2 trp $1-289$ ura3-52 & Invitrogen \\
\hline 614 & $\alpha$ leu23,112 ura3-52 bis3- $\Delta 200$ lys2-801 pep4-3 can1r & T. Stevens, University of Oregon \\
\hline 1308 & 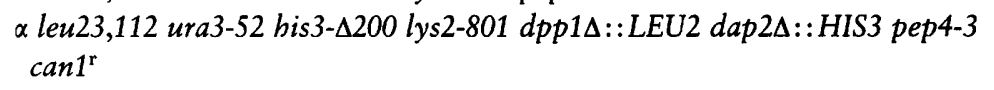 & T. Stevens, University of Oregon \\
\hline \multicolumn{3}{|c|}{ Y. lipolytica } \\
\hline CX161-1B & A ade1 & \\
\hline P17M & $A$ ade1 ura3- $\triangle E$ XPR2' (P17M AEP) & Yaver et al. (1992) \\
\hline SMY1 & $A$ ade 1 ura3- $\Delta E$ & This study \\
\hline SMY2 & $A$ ade1 ura3- $\Delta E \times$ xpr2- $\Delta A$ & This study \\
\hline SMA19V & $A$ ade1 ura3- $\triangle E$ XPR2' (A19V AEP) & This study \\
\hline SMS397A & $A$ ade1 ura3- $\triangle E$ XPR2' (S397A AEP) & This study \\
\hline SM $\triangle D P$ & $A$ ade1 ura3- $\triangle E$ XPR2' ( $\triangle \mathrm{DP}$ AEP) & This study \\
\hline DO613 & A ade1 xpr6-13 & Enderlin et al. (1993) \\
\hline
\end{tabular}

Mutagenesis Kit manual). Y. lipolytica was maintained on YM or $\mathrm{YM}^{+}$(Lee \& Ogrydziak, 1977) and S. cerevisiae on YPD (Sherman et al., 1986). Y. lipolytica competent cells were prepared in YPDC medium. The selective medium for transformants was YLT [SM (Sherman et al., 1986) plus $0.087 \%$ drop out mixtures] lacking a specific amino acid or base. YPGal is YPD with $2 \%(w / v)$ galactose substituted for $2 \%(w / v)$ glucose. GYX, GPP* and GC media were used for growing and labelling Y. lipolytica cells (Matoba et al., 1988; Yaver et al., 1992).

Strains. E. coli MV1190 and CJ236 were used for in vitro mutagenesis (Bio-Rad Muta-Gene M13 In vitro Mutagenesis Kit manual) and JM105 was used for DNA manipulations (Sambrook et al., 1989). Yeast strains used or constructed for this study are described in Table 1. SMY1 and SMY2 contain deletions of $U R A 3$ and of both $U R A 3$ and $X P R 2$, respectively, in a wild-type (CX161-1B $A$ ade1) background. A $0.4 \mathrm{~kb}$ EcoRV fragment from URA3 in pIMR41 was deleted to construct pIMR105 (ura3- $\Delta E$ flanked by SalI sites in pUC13). The Ncol site in ura3- $\Delta E$ in pIMR105 was destroyed by addition of BamHI linkers to form pIMR116. Filling in the BamHI site of pIMR107 containing the BamHI/SalI $4.3 \mathrm{~kb}$ ADE1 fragment in pBR322 yielded pIMR115. The $1.3 \mathrm{~kb}$ SalI fragment from pIMR 116 containing the modified $u$ ra3 $-\Delta E$ was integrated into the SalI site at the end of the ADE1 gene in pIMR115 to yield pIMR112. pIMR112 was linearized by cutting at the now unique Bam HI site to target to URA3, transformed into CX161-1B and $\mathrm{Ade}^{+}$colonies selected. SMY1 was obtained by selecting for $\mathrm{Ade}^{-} \mathrm{Ura}^{-}$loop-outs using 5FOA. The presence of the $u r a 3-\Delta E$ construct was confirmed by Southern blotting. To construct SMY2, a $0 \cdot 15 \mathrm{~kb} A p a \mathrm{I}$ fragment was deleted from XPR2 in pIMR100 (URA3 in SalI and XPR2 in EcoRI/HindIII sites of pBR322) to form pIMR122. pIMR122 was linearized with ClaI to target at $X P R 2$, transformed into SMY1 and $\mathrm{Ura}^{+}$colonies selected. SMY2 was obtained by selecting for $\mathrm{Xpr}^{-} \mathrm{Ura}^{-}$loop-outs using 5-FOA. The presence of the $x p r 2-\Delta A$ construct was confirmed by Southern blotting.

In vitro mutagenesis. The Bio-Rad Muta-Gene M13 In vitro Mutagenesis Kit, based on methods described by Kunkel et al. (1987), was used for site-directed mutagenesis. Construction of P17M has been described previously (Yaver et al., 1992). For SMA19V, primer 5' CTCCCCTGGTCGCCCCTGC 3' was used to convert $\mathrm{Ala}_{19}$ (GCC) to $\mathrm{Val}_{19}$ (GTC). For SM $\Delta \mathrm{DP}$, primer 5' TCACTGCCGTTCT GGCCCAGGTTCCTGCCG CCGCTGCCTA $3^{\prime}$ was used to remove the -X-Ala-, -XPro- dipeptide stretch from $\mathrm{Ala}_{16}$ to $\mathrm{Pro}_{33}$ and to insert CAGGTT encoding GIn-Val. For SMS397A, primer 5' CTCCGGT ACCGCCATGGCCT $3^{\prime}$ was used to change $\mathrm{Ser}_{397}$ (TCC) to $\mathrm{Ala}_{397}$ (GCC).

Site-directed mutagenesis was done in M13mp18 containing the $1.7 \mathrm{~kb} S p h \mathrm{I} / \mathrm{XbaI}$ XPR2 fragment. The mutagenized $S p h I / X b a I$ fragment was cloned into $S p h I-X b a I-d i g e s t e d$ pIMR100. The plasmids containing the mutations were cut with $M l u I$ to target upstream of $x p r 2-\Delta A$ in SMY2. $\mathrm{Ura}^{+}$ transformants were plated on 5-FOA medium to select for Ura ${ }^{-}$loop-outs. Replacement of $x p r 2-\Delta A$ by the mutagenized version of XPR2 was confirmed by Southern blotting.

Protease assays. Skim milk (SKM) plates for detection of production of AEP by Y. lipolytica were prepared as described previously (Ogrydziak \& Mortimer, 1977; Enderlin \& Ogrydziak, 1993). SKM plates for detection of production of AEP by $S$. cerevisiae were prepared in a similar manner except for the addition of $0.1 \%$ peptone, $50 \mathrm{mg}$ His and Lys $1^{-1}, 100 \mathrm{mg}$ Leu $1^{-1}$, and $1 \%(\mathrm{w} / \mathrm{v})$ either galactose or glucose. Extracellular protease activity secreted by $S$. cerevisiae strains was measured using the $A_{750}$ casein hydrolysis method (Ogrydziak \& Scharf, 1982).

Pulse-chase immunoprecipitation. Pulse-chase labelling with $\left[{ }^{3} \mathrm{H}\right]$ Leu, disruption of cells, immunoprecipitation of cell extracts with AEP antibody, SDS-PAGE of immunoprecipitates on $10-15 \%(\mathrm{w} / \mathrm{v})$ gradient gels and fluorography was based on previously described procedures (Matoba et al., 1988).

S. cerevisiae strains 614 and 1308 were grown to midexponential phase in synthetic minimal medium $(0 \cdot 67 \%$ yeast

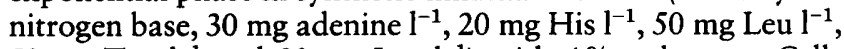
$50 \mathrm{mg} \mathrm{Trp}^{-1}$ and $20 \mathrm{mg} \mathrm{Lys}^{-1}$ ) with $4 \%$ galactose. Cells were harvested by centrifugation $(5000 \mathrm{~g}$ for $5 \mathrm{~min}$ ) and resuspended in $0.25 \mathrm{ml}$ of the same medium. A 2.5 min pulse with $1.02 \times 10^{7}$ Expre $^{35}{ }^{35} \mathrm{~S}$ label (NEN) was followed by the addition of unlabelled Met and Cys to final concentrations of $50 \mathrm{mM}$ and $25 \mathrm{mM}$, respectively, and addition of $0.2 \mathrm{ml}$ fresh medium with yeast extract to $0 \cdot 2 \%$. At various times after initiation of chase, $0.1 \mathrm{ml}$ samples were removed and cells quickly separated from the medium by a pulse spin in a 
microcentrifuge. The supernatant was then precipitated with TCA ( $10 \%$ final concentration).

Extracellular AEP was immunoprecipitated essentially as described by Harding et al. (1995) using $5 \mu$ l AEP antibody and a $40 \%(\mathrm{v} / \mathrm{v})$ slurry of Protein A-Sepharose. Immunocomplexes were washed twice with Tween-20 immunoprecipitation buffer and solubilized in $50 \mu \mathrm{l}$ Laemmli sample buffer. Samples were resolved on a $10 \%(w / v)$ SDS-PAGE gel. Quantification was done with phosphorimage analysis using the Fuji FUJIX BAS1000 Bioimaging Analyzer (Fuji Medical Systems).

Endoglycosidase $\mathbf{H}$ digestion. Immunoprecipitated samples were digested with endoglycosidase $\mathrm{H}$ as described previously by Yaver et al. (1992).

Radiosequencing. Radiolabelled cell extracts were prepared as described for pulse-chase immunoprecipitation with the following modifications: $40 \mathrm{ml}$ cells were labelled with $9.25 \times 10^{7} \mathrm{~Bq}\left[{ }^{3} \mathrm{H}\right]$ Leu and $3.7 \times 10^{7} \mathrm{~Bq}\left[{ }^{35} \mathrm{~S}\right]$ Met. Six millilitre samples were taken at 2 and 4 min and cell extracts immunoprecipitated. The immunoprecipitates were combined and run on a $10-15 \%$ (w/v) SDS-PAGE gel (Hunkapiller et al., 1983). The gel was dried, the position of the $55 \mathrm{kDa}$ band located by autoradiography and the band cut out. The labelled polypeptide was electroeluted in Tris/glycine buffer $(0.5 \times$ Laemmli running buffer) in a Sample Concentrator Model 1750 (ISCO), the sample electrodialysed in $10 \mathrm{mM} \mathrm{NH}_{4} \mathrm{HCO}_{3}$, $0.02 \%$ SDS buffer and lyophilized. The sample was resuspended, $5 \mathrm{nmol}$ apomyoglobin added and automated Edman degradation and counting of radioactivity in each cycle were done as described previously (Yaver et al., 1992).

DPAPase inhibitor. The DPAPase inhibitor Pro-boroPro, where boroPro is the $\alpha$-amino boronic acid analogue of proline, was obtained from W. W. Bachovchin (Tufts University, Boston, MA, USA) (Flentke et al., 1991). Pro-boroPro was added at a final concentration of $0.2 \mathrm{mM}$ when cells were transferred to GC medium as in a standard pulse-chase immunoprecipitation experiment (Matoba et al., 1988). After $20 \mathrm{~min}, \mathrm{PMSF}$ was added ( $2 \mathrm{mM}$ final concentration). After an additional $10 \mathrm{~min}$ the cells were labelled for $60 \mathrm{~s}$, the chase added and extracellular samples taken after 10 and $20 \mathrm{~min}$ were TCA-precipitated. Samples were resuspended in Laemmli sample buffer.

GAL1-XPR2 fusion. An oligonucleotide $\left(5^{\prime}\right.$ CCCACTATCCAAGCTTCCAATCCAA $3^{\prime}$ was used to create a HindIII site (centred 14 bp upstream of the XPR2 translation initiation site ATG) in pIMR122 to yield pGX-2. A version encoding inactive AEP was constructed in case production of active AEP was lethal for $S$. cerevisiae. The HindIII/XbaI fragment of pIMR119 (S397A version of XPR2 in pIMR100) was replaced with the HindIII/XbaI fragment from pGX-2 to form pIMR87. The HindIII/EcoRI fragments from pGX-2 and pIMR87 were inserted into the polylinker site of pYES2 (an $S$. cerevisiae $2 \mu$ expression plasmid with the GAL1 promoter and $U R A 3$; Invitrogen) downstream of the GAL1 promoter to create pIMR 88 and pIMR 89 , respectively.

DNA sequencing. Single- and double-stranded DNA sequencing was done by the dideoxy method using the Sequenase Kit (USB) and $\left[{ }^{35} \mathrm{~S}\right] \mathrm{dATP}$.

Protease protection. The protocol for spheroplast preparation, lysis and proteinase $\mathrm{K}$ treatment was essentially that followed by Lee \& Ogrydziak (1997). Half the volume of cells were processed and $200 \mu \mathrm{l}$ cell extract was used for proteinase $\mathrm{K}$ (AMRESCO) treatments. For Western blotting, pellets were resuspended in $100 \mu \mathrm{l} \mathrm{Laemmli}$ buffer, neutralized with $1 \mathrm{M}$
Tris base and analysed by SDS-PAGE. Western blotting was done as described previously (Matoba et al., 1988). AEP antibodies were used at a 1:1000 dilution; proteins bound to nitrocellulose were detected using the ECL Western Blotting Detection Kit (Amersham) and horseradish-peroxidaselabelled second antibody (1:20000) (Accurate Chemicals).

\section{RESULTS AND DISCUSSION}

\section{Signal peptide cleavage site}

AEP is cleaved at several sites within the $\mathrm{N}$-terminal prepropeptide, but the location and function of some of these processing events has not been determined. Most likely the largest AEP precursor $(55 \mathrm{kDa})$ detected in cell extracts in pulse-chase immunoprecipitation experiments lacks the signal peptide (Matoba et al., 1988; Fabre et al., 1991). The predicted site of cleavage is after $\mathrm{Ala}_{15}(S$ value is 7.0) (von Heijne, 1986) and this has been shown to be the cleavage site in the P17M version of XPR2 (Yaver et al., 1992). To determine if this was also the cleavage site for wild-type AEP, N-terminal amino acid radiosequencing of immunoprecipitated $\left[{ }^{3} \mathrm{H}\right] \mathrm{Leu}-$ and $\left.{ }^{35} \mathrm{~S}\right]$ Met-labelled $55 \mathrm{kDa}$ AEP precursor was done. Leu is predicted at positions 3 and 10 and Met at position 1 for an AEP precursor still containing the signal peptide, and only Leu at position 3 and no Met for a precursor cleaved after $\mathrm{Ala}_{15}$. The highest ${ }^{3} \mathrm{H}$ counts were in cycle 3 with only background ${ }^{3} \mathrm{H}$ counts in cycle 10 (Fig. 2) and ${ }^{35} \mathrm{~S}$ counts in all cycles including cycle 1 were at background (not shown), suggesting that the wild-type $55 \mathrm{kDa}$ AEP precursor lacks the signal peptide.

\section{Role of AEP activity in AEP processing}

Based on sequence homology, AEP is a subtilisin-like protease with $\mathrm{Ser}_{397}$ as part of the active site triad (Davidow et al., 1987). Two putative intracellular precursors of 44 and $36 \mathrm{kDa}$ have been seen in many pulse-chase immunoprecipitation experiments (Matoba

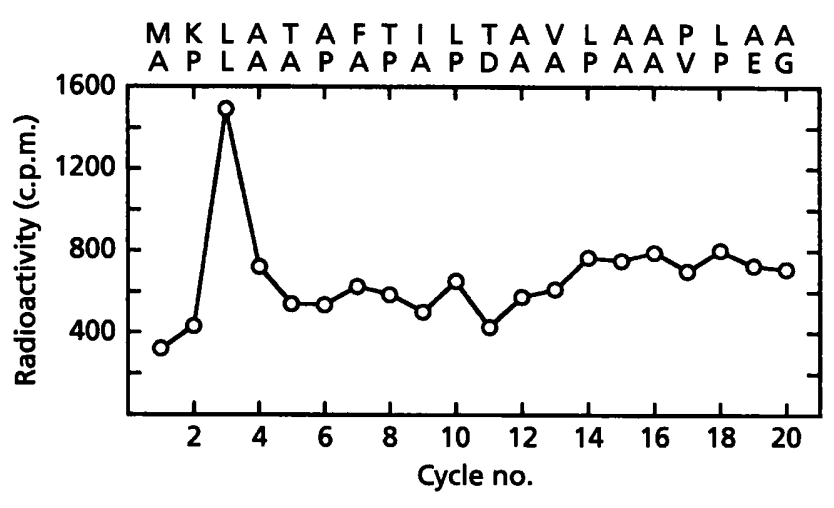

Fig. 2. The $55 \mathrm{kDa}$ AEP precursor lacks the signal peptide and cleavage occurs at the predicted site after Ala ${ }_{15}$. Radiosequencing of $55 \mathrm{kDa}$ AEP precursor; $\left[{ }^{3} \mathrm{H}\right]$ Leu counts per cycle. The upper sequence starts at Met $_{1}$ and the lower at $\mathrm{Ala}_{16}$, the first amino acid after the predicted signal peptide cleavage site. 


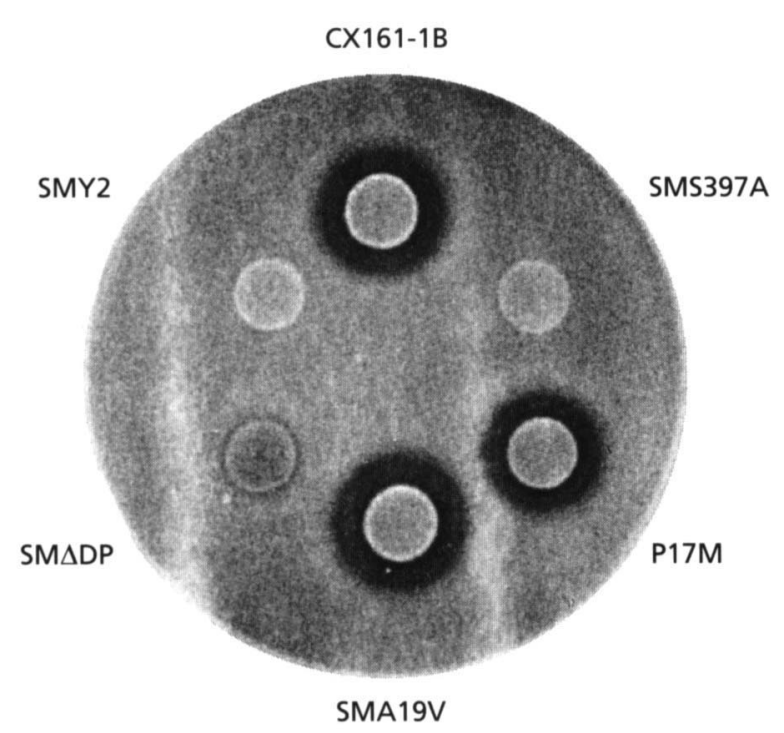

Fig. 3. Production of AEP activity on SKM plates by $Y$. lipolytica strains. Cells growing exponentially in YPD at $23^{\circ} \mathrm{C}$ were diluted and $10^{5}$ cells in $10 \mu \mathrm{l}$ inoculated onto plates which were incubated for $40 \mathrm{~h}$ at $23^{\circ} \mathrm{C}$. Secretion of active AEP is detected as a halo.

et al., 1988). The $44 \mathrm{kDa}$ polypeptide contains about $2 \mathrm{kDa}$ of $\mathrm{N}$-linked carbohydrate and is of the appropriate size to result from cleavage after $\mathrm{Lys}_{59}-\mathrm{Arg}_{60}$ (Matoba et al., 1988). The $36 \mathrm{kDa}$ polypeptide contains no $N$-linked carbohydrate and there is no potential dibasic cleavage site appropriately located downstream of the glycosylation site at $\mathrm{Asn}_{123}$ (Matoba et al., 1988). The possibility that AEP activity was needed for AEP processing and was involved in producing one or more of these precursors was investigated by changing $\mathrm{Ser}_{397}$ to Ala (S397A). As for SMY2, a strain containing a deletion of the XPR2 gene which encodes AEP, SMS397A, produced no zone of clearing on a SKM plate (Fig. 3). Processing of AEP in pulse-chase immunoprecipitation experiments was similar to wild-type except that less mature AEP was secreted and little or none of the 44 and $36 \mathrm{kDa}$ precursors was present (Fig. 4a). The production of mature AEP implies that AEP activity was not necessary for processing of AEP precursors. The absence of the 44 and $36 \mathrm{kDa}$ precursors suggests that they may be degradation products produced by AEP activity during sample preparation and not in vivo AEP processing intermediates.

The fact that no labelled AEP precursor accumulated in the extracellular medium when PMSF, which inhibits AEP activity, was included during labelling of wild-type cells is also consistent with AEP activity not being needed for AEP processing (Matoba et al., 1988). It also suggests that AEP activity is not necessary for extracellular processing of AEP, i.e. no AEP precursor is secreted which is quickly proteolytically processed by AEP activity.

\section{Role of -X-Ala-, -X-Pro- dipeptide stretch in AEP processing}

For DPAPase to remove a dipeptide, it requires a free $\mathrm{N}$ terminus and either Ala or Pro as the second amino acid (Kreil, 1990). The P17M and A19V mutations were constructed either to completely prevent DPAPase processing (P17M) or to allow removal of only the $\mathrm{Ala}_{16}-\mathrm{Pro}_{17}$ dipeptide (A19V) (Fig. 1b). If removal of the dipeptide stretch is needed for KEX2-like processing to occur, then it would be predicted that an AEP precursor(s) of molecular mass identical (P17M) or very similar (A19V) to the wild-type $55 \mathrm{kDa}$ AEP precursor would be secreted. For P17M, the unexpected result of posttranslational translocation was obtained (Yaver et al., 1992). Also production of AEP activity was reduced compared to wild-type (Fig. 3). For strain SMA19V, production of AEP activity (SKM zones) (Fig. 3) and AEP processing and secretion kinetics (Fig. 4b) were very much like wild-type (Matoba et al., 1988; see Figs 1 and 5). The A19V $55 \mathrm{kDa}$ AEP precursor had mobility on SDS-PAGE and $N$-linked carbohydrate content indistinguishable from the wild-type $55 \mathrm{kDa}$ AEP precursor (data not shown). As expected, the $52 \mathrm{kDa}$ precursor, a product of DPAPase processing, was not detected.

The role of the dipeptide stretch was also investigated by deleting the region encoding the dipeptides. In SM $\triangle \mathrm{DP}$ the nine dipeptides starting with $\mathrm{Ala}_{16}$ were deleted and Gln-Val inserted (Fig. 1b). Based on $S$ values (von Heijne, 1986), signal peptide cleavage is much more likely to occur after $\mathrm{Ala}_{15}$ (as in wild-type) if $\mathrm{Ala}_{15}$ is followed by Gln-Val than if it was followed by Glu-Gly as would occur without the insertion. If the dipeptide stretch functions to delay AEP activation by the XPR6 processing endoprotease, then AEP might be activated prematurely in SM $\Delta D P$ and the cells might be sick or unviable. In pulse-chase immunoprecipitation experiments with SM $\triangle \mathrm{DP}$, only one AEP precursor (ca $51 \mathrm{kDa}$ ) was detected intracellularly (Fig. 4c). No mature AEP or polypeptides in the $50-55 \mathrm{kDa}$ range were detected extracellularly. The material at $65 \mathrm{kDa}$ is most likely also secreted by other strains but not detected because the fluorographs were only exposed for $3 \mathrm{~d}$ as opposed to $15 \mathrm{~d}$ for SM $\Delta \mathrm{DP}$. There was very weak clearing on the skimmed milk plate (Fig. 3), indicating that a very low level of AEP activity was secreted. These results suggest that most of the $\triangle \mathrm{DP}$ precursor had never reached the late Golgi where XPR6 processing presumably occurs. We suspected that it was misfolded and retained in the ER. Surprisingly, the mobility of the $\triangle D P A E P$ precursor did not change after treatment with endoglycosidase $H$, indicating that, unlike other translocated AEP precursors, it did not contain $\mathrm{N}$-linked carbohydrate (Fig. 5a). Folding and glycosylation reactions can compete in vivo and, in some cases, slowing folding can increase the level of glycosylation (Holst et al., 1996). In this case, we suspect altered folding makes the normal $\mathrm{N}$-linked glycosylation site (Asn ${ }_{123}$ in the wild-type pro-region) unavailable. 
(a)
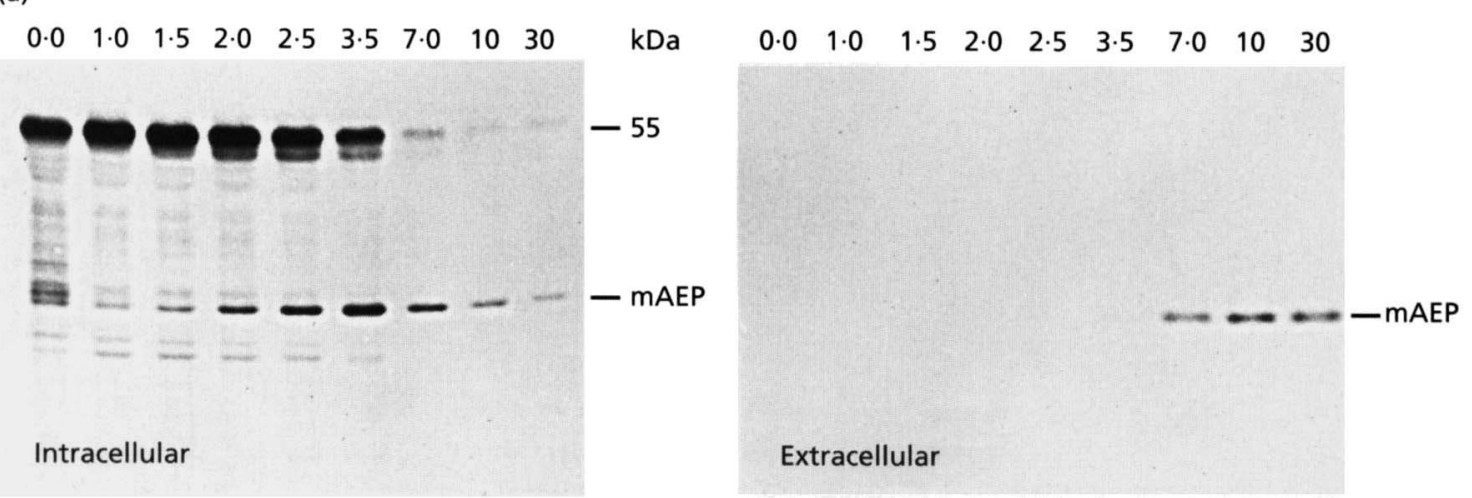

(b)

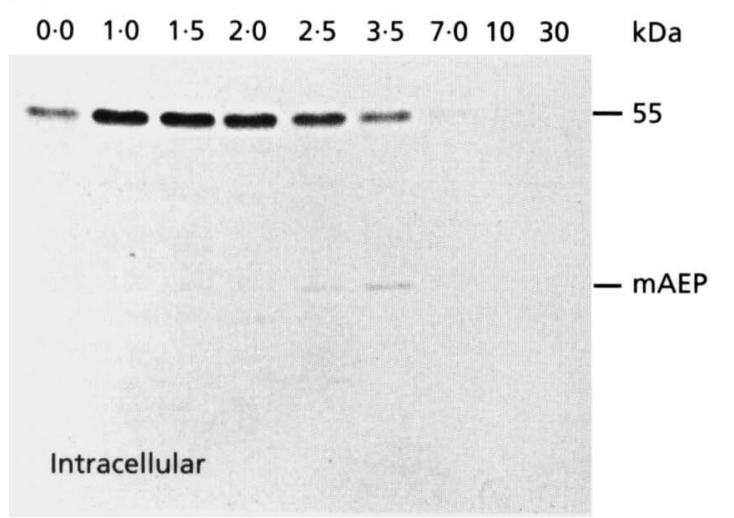

$\begin{array}{llllllllll}0.0 & 1.0 & 1.5 & 2.0 & 2.5 & 3.5 & 7.0 & 10 & 30 & \mathrm{kDa}\end{array}$

(c)
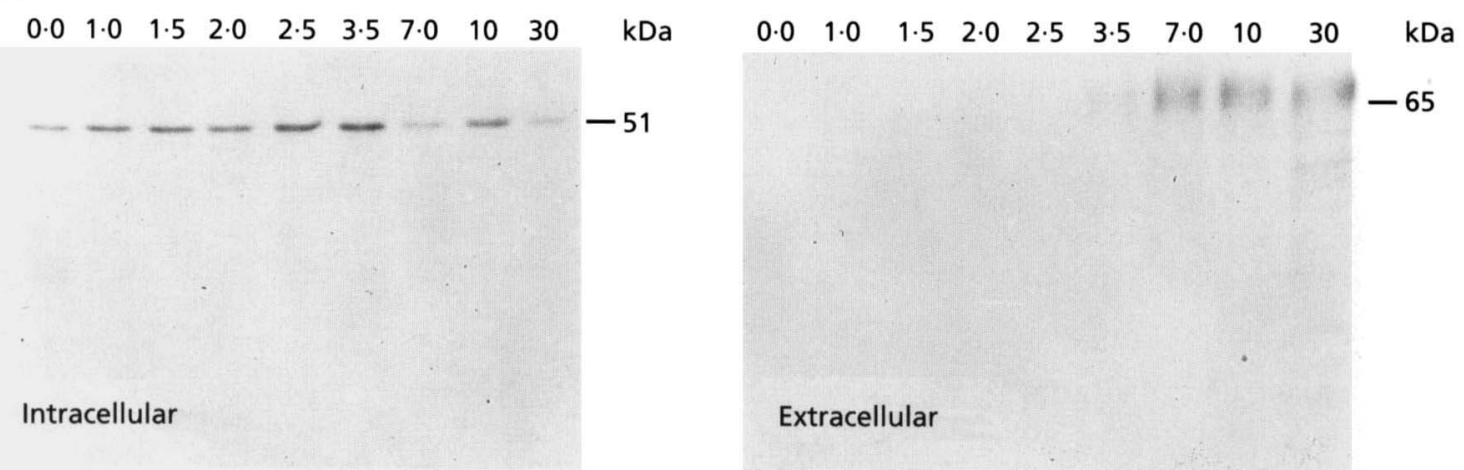

Fig. 4. (a) Mature AEP is secreted by SMS397A. (b) SMA19V behaves much like wild-type, except that no $52 \mathrm{kDa}$ AEP precursor was detected. (c) For SM $\triangle D P$ only a $51 \mathrm{kDa}$ AEP precursor is detected which is not processed further, and little or no mature AEP is secreted. Pulse-chase immunoprecipitation was done by labelling each of the strains with [ $\left.{ }^{3} \mathrm{H}\right] \mathrm{Leu}$ for $45 \mathrm{~s}$. Cell extracts were immunoprecipitated and supernatant samples TCA-precipitated. The precipitates were analysed by SDS-PAGE and fluorography. The position of mature AEP (mAEP) and estimated molecular masses of AEP precursors and pro-region, and of some secreted proteins are indicated. The time after chase $(\mathrm{min})$ is shown above each lane.

The deletion of 16 aa could account for an approximately $2 \mathrm{kDa}$ decrease in size. Because both a $55 \mathrm{kDa}$ translocated AEP precursor lacking both the 16 aa and the $2 \mathrm{kDa}$ of $\mathrm{N}$-linked carbohydrate and a $53 \mathrm{kDa}$ untranslocated (and therefore unglycosylated) AEP precursor lacking the 16 aa would be expected to have mobilities similar to that of the $\triangle \mathrm{DP}$ AEP precursor, a protease protection experiment was done to determine if the SM $\triangle \mathrm{DP}$ AEP precursor was translocated. The SM $\triangle D P$ AEP precursor was not degraded by proteinase $\mathrm{K}$ in the absence of Triton X-100 (Fig. 5b, lane 2) but it was in its presence (Fig. 5b, lane 3), suggesting that the precursor had been translocated into a membraneenclosed compartment. These results suggest that the dipeptide stretch is necessary for folding into a form competent for translocation out of the ER. 
(a)

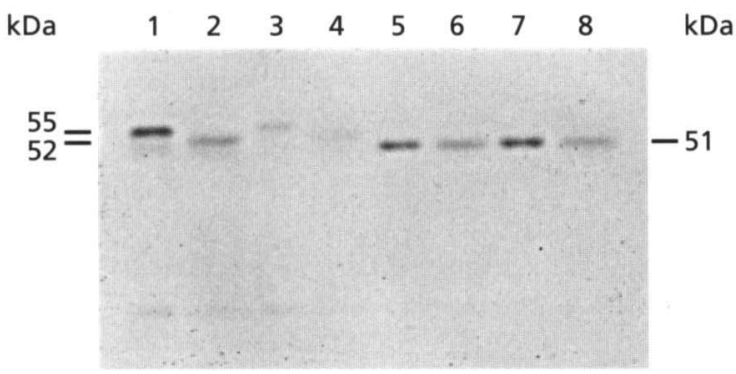

(b)

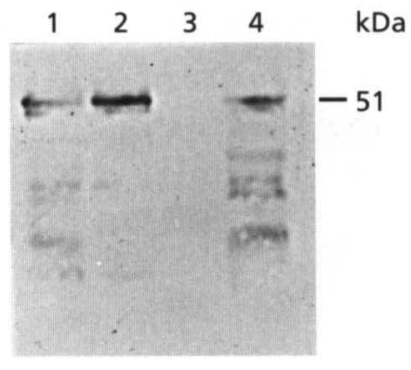

Fig. 5. (a) The SM $\triangle D P 51 \mathrm{kDa}$ AEP precursor does not contain $\mathrm{N}$-linked carbohydrate. ${ }^{3} \mathrm{H}$-Labelled immunoprecipitated intracellular samples from wild-type (lanes 1-4) and SM $\triangle D P$ (lanes 5-8) were taken either 3 min (lanes 1, 2, 5 and 6) or 5 min (lanes 3, 4, 7 and 8) after addition of the chase. Samples in lanes 2, 4, 6 and 8 were incubated with endoglycosidase H. (b) The SM $\triangle D P 51 \mathrm{kDa}$ AEP precursor is enclosed in a membrane-bound compartment. Spheroplasts were prepared, lysed and either subjected to a mock protease treatment (lane 1) or incubated with proteinase $K$ in the absence (lane 2) or presence (lane 3) of Triton X-100. Lane 4, Triton X-100 with no proteinase K. After Western blotting, the SM $\Delta D P$ $51 \mathrm{kDa}$ AEP precursor was visualized using antiserum to AEP and chemiluminescence.

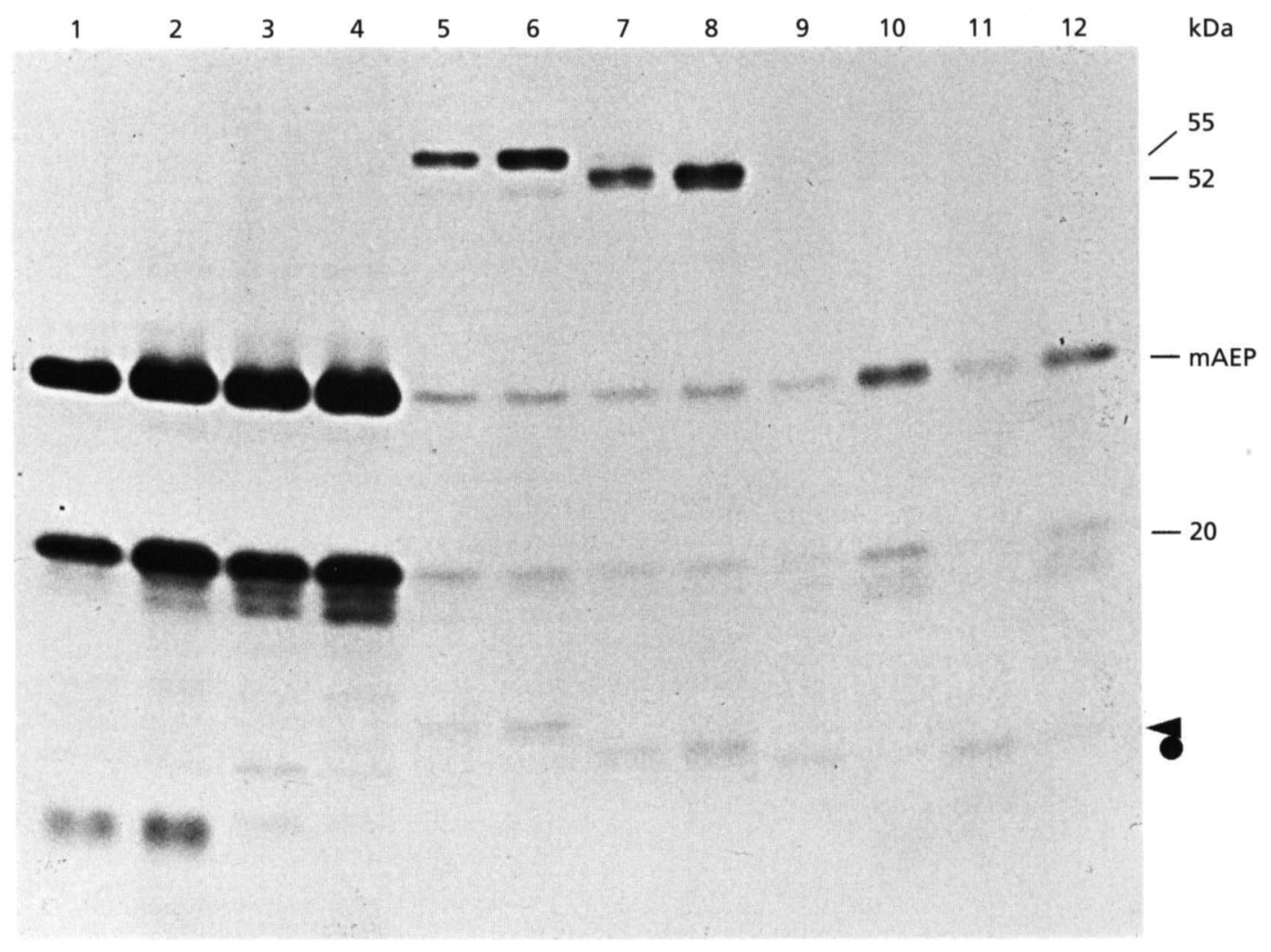

Fig. 6. AEP is processed and mature AEP secreted in the presence of Pro-boroPro. Cells were labelled with [ $\left.{ }^{3} \mathrm{H}\right] \mathrm{Leu}$ either in the presence (lanes 1, 2, 5, 6, 9 and 10) or absence (lanes 3, 4, 7, 8, 11 and 12) of Pro-boroPro. Supernatant samples were collected 10 min (lanes 1, 3, 5, 7, 9 and 11) and 20 min (lanes 2, 4, 6, 8, 10 and 12) after the start of labelling and TCA-precipitated. Twenty microlitres of resuspended sample (equivalent to $60 \mu$ l extracellular medium) were applied per lane. Lanes: 1-4, wild-type (CX161-1B) samples; 5-8, D0613; 9-12, P17M. The positions of the 55 and 52 kDa AEP precursors, mature AEP (mAEP) and the $20 \mathrm{kDa}$ AEP pro-region are indicated. The filled arrowhead and circle indicate putative AEP propeptide fragments which possibly differ in the number of dipeptides retained.

\section{Role of DPAPase activity in AEP processing}

Results with strain A19V suggest that removal of the dipeptide stretch is not needed for AEP processing by Xpr6p. To eliminate the possibility that the A19V mutation caused an altered conformation of AEP, we examined the susceptibility to Xpr6p processing of wildtype protein using the DPAPase inhibitor Pro-boroPro (Flentke et al., 1991). If removal of the dipeptide stretch is required for XPR6 processing, then inhibition of 
(a)

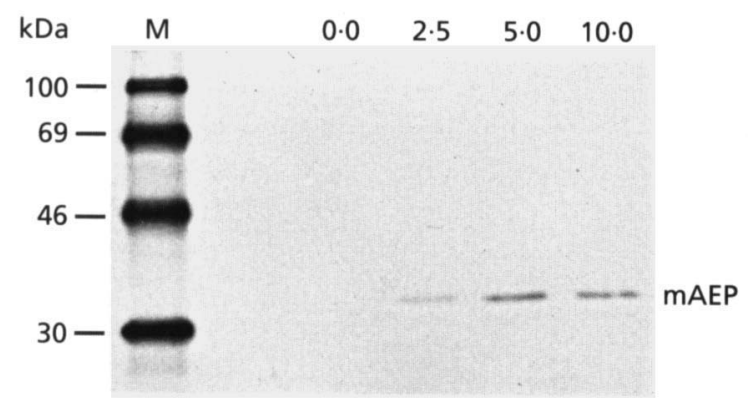

(b)
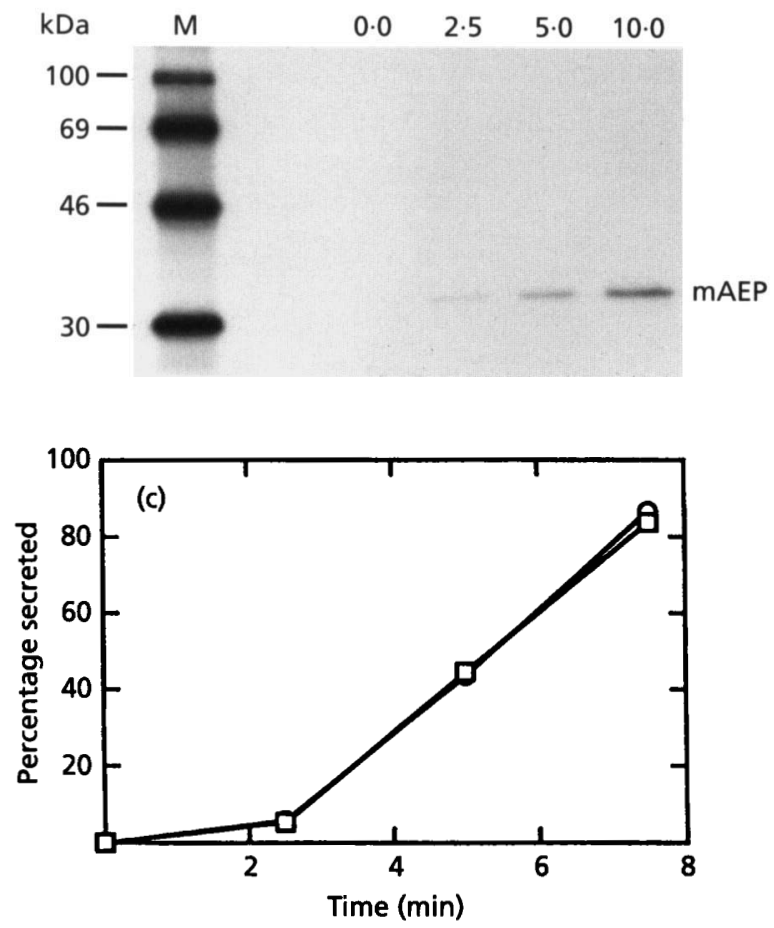

Fig. 7. (a, b) Only mature AEP and no AEP precursors are secreted by $S$. cerevisiae strains [a, 614(pIMR88); b, 1308(pIMR88)] whether or not they produce DPAPase activity. Pulse-chase labelling was done for $2.5 \mathrm{~min}$ with [35]Met and $\left.{ }^{35} \mathrm{~S}\right]$ Cys and the supernatant samples were TCA-precipitated, resolubilized, immunoprecipitated with antiserum to AEP, analysed by SDS-PAGE and detected by phosphorimaging. The time after chase ( $\mathrm{min}$ ) is shown above each lane. The position of mature AEP (mAEP) is indicated. The molecular masses of ${ }^{14} \mathrm{C}$ methylated standards (Amersham) phosphorylase b (100 kDa), bovine serum albumin $(69 \mathrm{kDa})$, ovalbumin $(46 \mathrm{kDa})$ and carbonic anhydrase ( $30 \mathrm{kDa}$ ) are indicated (lane $M)$. (c) Kinetics of AEP secretion and levels of AEP secreted are similar for the two strains. Results of quantification by phosphorimaging analysis of label incorporated into mature AEP immunoprecipitated from supernatant samples were normalized with the highest incorporation at $7.5 \mathrm{~min}$ in a given experiment taken as $100 \%$. Results from four separate experiments were averaged. $\square, 614$ (pIMR88); 0,1308 (pIMR88).

DPAPase activity should result in secretion of a $55 \mathrm{kDa}$ AEP precursor. For three strains Pro-boroPro was added before labelling in a pulse-chase immunoprecipitation experiment and extracellular samples TCA-precipitated 10 and $20 \mathrm{~min}$ after the chase. PMSF was also added before labelling to stabilize the secreted AEP propeptide (Matoba \& Ogrydziak, 1989).

Although it is not known which intracellular compartments of the secretory pathway are accessible to this inhibitor, results with strain DO613 indicate that ProboroPro did inhibit DPAPase activity in vivo (Fig. 6, lanes $5-8)$. xpr6-13 is a leaky mutation in the gene encoding XPR6, the KEX2-like processing endoprotease responsible for the cleavage after Lys-Arg at the end of the AEP propeptide (Enderlin \& Ogrydziak, 1993). Strain DO613 secretes a $52 \mathrm{kDa}$ AEP precursor which has undergone DPAPase processing (Matoba \& Ogrydziak, 1989). As expected if the dipeptide stretch was not removed in the presence of Pro-boroPro, the larger $55 \mathrm{kDa}$ AEP precursor was secreted (Fig. 5, lanes 5-6).

Even when DPAPase activity was inhibited by ProboroPro, there was no evidence for secretion of a $55 \mathrm{kDa}$ AEP precursor by the wild-type, providing additional evidence that removal of the dipeptide stretch was not necessary for KEX2-like processing of AEP (Fig. 6, lanes $1-2)$.

\section{Secreted AEP propeptide}

Several results are consistent with the $19-20 \mathrm{kDa}$ doublet secreted by wild-type being the AEP propeptide: (i) its secretion kinetics were similar to AEP; (ii) it is near the expected size; (iii) like the 55 and $52 \mathrm{kDa}$ AEP precursors, it contains about $2 \mathrm{kDa}$ of $\mathrm{N}$-linked carbohydrate; and (iv) it lacks Trp, Met and Cys as predicted for the AEP propeptide (Matoba et al., 1988). Originally, we proposed that the $19-20 \mathrm{kDa}$ doublet contained the intact propeptide with the $20 \mathrm{kDa}$ form retaining some dipeptides and the $19 \mathrm{kDa}$ form devoid of dipeptides (Matoba et al., 1988). Results obtained for SMA19V and $\mathrm{P} 17 \mathrm{M}$ and for all strains tested with Pro-boroPro are most consistent with both the 19 and $20 \mathrm{kDa}$ bands being derived from the AEP propeptide. One interpretation is that since the mobility of the major secreted form of the AEP propeptide is unaffected in P17M and by treatment with Pro-boroPro, in all cases it contains the dipeptide stretch. For DO613, Pro-boroPro inhibition resulted in accumulation of a precursor larger than the $52 \mathrm{kDa}$ precursor which lacks most of the dipeptides (Matoba \& Ogrydziak, 1989). If this mobility difference reflects the extent of removal of the dipeptide stretch, then the major secreted propeptide synthesized in the presence of Pro-boroPro (Fig. 6, lanes 5-6) should be larger than in its absence (Fig. 6, lanes 7-8). In fact, they have similar mobilities and this is consistent with the possibility that the $20 \mathrm{kDa}$ bands contain little or none of the dipeptide stretch. In the $13-17 \mathrm{kDa}$ region a larger band is found in the Pro-boroPro-treated sample (Fig. 6, lanes 5-6) compared to the untreated sample (Fig. 6, lanes 7-8), suggesting that these bands differ in the number of -X-Ala-, -X-Pro- dipeptides they contain. Since these bands were precipitated by TCA and not by antibodies to the AEP pro-region, however, it is not certain that they are AEP-related. The first model in which the $20 \mathrm{kDa}$ propeptides contain the dipeptide 
stretch can be reconciled with the DO613 results if the $55 \mathrm{kDa}$ precursor is a better substrate than the propeptide for DPAPase processing and if for wild-type AEP, Xpr6p cleavage occurs first and the propeptide is not processed by DPAPase.

\section{Processing of AEP in S. cerevisiae}

Mutant strains lacking DPAPase activity are not available for Y. lipolytica. To determine if DPAPase processing was necessary prior to Kex $2 p$ cleavage without relying on the chemical inhibitor Pro-boroPro, we expressed AEP in S. cerevisiae. S. cerevisiae INV Sc1 transformed with pIMR88 produced small zones of clearing on Gal-SKM plates. Western blots of supernatant medium from YPGal-grown cells probed with AEP antibody revealed a single band of the same mobility as mature AEP (data not shown).

To examine the role of DPAPase activity in AEP processing, the multicopy $2 \mu$ plasmids pIMR 88 (wildtype XPR2) and pIMR89 ( $x p r 2$ with the S397A mutation) were transformed into $S$. cerevisiae strain 1308 containing disrupted copies of both DPP1 (STE13, encoding the Golgi DPAP A) and DAP2 (encoding vacuolar DPAP B), and the related strain 614, wild-type for both DPAPase activities. No zones of clearing were detected for any of the transformants grown on GluSKM plates. On Gal-SKM plates, clearing was detected for both 614 and 1308 with pIMR88 but not with pIMR89, suggesting that, at least in $S$. cerevisiae, DPAPase processing is not required for production of AEP activity (data not shown). Clearing was only detected around areas of heavy growth and not around single colonies, and zones of clearing were significantly larger for cultures incubated at $23^{\circ} \mathrm{C}$ than at $30^{\circ} \mathrm{C}$.

To determine if a kinetic defect in AEP secretion was occurring in the DPAP mutant strain, radiolabelled pulse-chase immunoprecipitations were performed. Cells were labelled for $2.5 \mathrm{~min}$, the label chased and samples of the extracellular medium taken at various times and immunoprecipitated with AEP antibody. The major band detected migrates with the same mobility on SDS-PAGE as mature AEP produced by Y. lipolytica (Fig. 7a). There was no delay in appearance of mature AEP in 1308 (pIMR88) versus 614(pIMR88) (Fig. 7b) and no evidence for secretion of AEP precursors in either strain (Fig. 7a). Therefore, it seems that a lack of DPAPase activity does not affect transit time or delay KEX2 processing of AEP. Preliminary results of immunoprecipitation of labelled cell extracts reveal that, unlike the case for Y. lipolytica, in S. cerevisiae AEP precursor(s) of about $55 \mathrm{kDa}$ are still cell-associated 15-30 min after the start of the chase (data not shown). If AEP precursors were trapped in the cell wall, some leakage into the extracellular medium might be expected. This was not found, suggesting that the AEP precursor(s) are intracellular and that their movement through the secretory pathway is slowed in S. cerevisiae whether or not DPAPase activity is present.

The immunoprecipitation experiments suggested that the levels of AEP secreted by the $S$. cerevisiae strains were comparable. This was confirmed by assaying AEP activity of the supernatant medium from 614(pIMR88) and 1308(pIMR88) grown in synthetic minimal medium containing $0.1 \%$ bovine serum albumin and either $2 \%$ glucose or $4 \%$ galactose. As expected no AEP activity was detected during growth on glucose. With galactose the growth rates were similar and AEP activity was almost identical $-5 \cdot 1 \pm 0.4 \mathrm{U}$ (mg dry wt cells) ${ }^{-1}$ for 614(pIMR88) and 5.3 $\pm 0.2 \mathrm{U}$ (mg dry wt cells) ${ }^{-1}$ for 1308(pIMR88) (means \pm SEM, $n=4$ ). In GPP medium $Y$. lipolytica produces $135 \mathrm{U}$ (mg dry wt cells) ${ }^{-1}$ (Ogrydziak $\&$ Scharf, 1982). Thus, much less AEP was secreted by $S$. cerevisiae strains with multiple copies of XPR2 than by a Y. lipolytica strain with a single copy. These results, however, confirm that DPAPase processing is not required for maturation and secretion of the enzyme.

We have investigated the role of the AEP propeptide and DPAPase processing on the biosynthesis of this secreted protein. Mutations that block removal of the dipeptide stretch, inhibition of DPAPase activity or use of strains that lack DPAPase activity all allowed secretion of mature-sized AEP. Removal of the dipeptide stretch is apparently not a prerequisite for proteolytic processing by Xpr6p which generates the mature AEP protein, even though DPAPase processing of AEP normally occurs in vivo (Matoba et al., 1989). If only the conformation of this region was important, however, it is unclear why the amino acid sequence has been constrained to maintain this stretch of amino acids as a substrate for DPAPase processing. The dipeptide stretch of AEP does appear to be needed for proper folding and transit. Constructs lacking this part of the propeptide are not able to exit from the ER and are not properly glycosylated. The dipeptide stretch may interact with machinery in the ER lumen such as chaperones to allow correct folding. Alternatively, it may be involved in transit out of the ER through interactions with proteins involved in vesicle packaging and cargo selection.

These data support previous studies on the importance of the dipeptide stretch for secretion of heterologous proteins (Tharaud et al., 1992). In addition, these results show that studies in non-conventional yeasts can take advantage of the well-developed cell biology and genetics (especially the availability of mutants) of $S$. cerevisiae.

\section{ACKNOWLEDGEMENTS}

We thank S. Nothwehr and T. Stevens (University of Oregon, Eugene, OR, USA) for generously supplying S. cerevisiae strains 614 and 1308 and W. W. Bachovchin for the ProboroPro. K.K. was partially supported by Pfizer Inc. This work was supported by the National Science Foundation and the California Agricultural Experiment Station.

\section{REFERENCES}

Davidow, L. S., O’Donnell, M. M., Kaczmarek, F. S., Pereira, D. A., Dezeeuw, J. R. \& Franke, A. E. (1987). Cloning and sequencing of the alkaline protease gene of Yarrowia lipolytica. J Bacteriol 169, 4621-4629. 
Enderlin, C. S. \& Ogrydziak, D. M. (1993). Cloning, nucleotide sequence, and functions of XPR6 which codes for a dibasic processing endoprotease from the yeast Yarrowia lipolytica. Yeast 10, 67-79.

Fabre, E., Nicaud, J. M., Lopez, M. C. \& Gaillardin, C. (1991). Role of the proregion in the production and secretion of the Yarrowia lipolytica alkaline extracellular protease. J Biol Chem 266, 3782-3790.

Fabre, E., Tharaud, C. \& Gaillardin, C. (1992). Intracellular transit of a yeast protease is rescued by trans-complementation with its prodomain. J Biol Chem 267, 15049-15055.

Flentke, G. R., Munoz, E., Huber, B. T., Plaut, A. G., Kettner, C. A. \& Bachovchin, W. W. (1991). Inhibition of dipeptidyl aminopeptidase IV (DP-IV) by Xaa-boroPro dipeptides and use of these inhibitors to examine the role of DP-IV in T-cell function. Proc Natl Acad Sci USA 88, 1556-1559.

Franke, A. E., Kaczmarek, F. S., Eisenhard, M. E., Geoghehan, K. F., DeZeeuw, J. R., O'Donnell, M. M., Gollaher, M. G. \& Davidow, L. S. (1988). Expression and secretion of bovine prochymosin by Yarrowia lipolytica. Dev Ind Microbiol 29, 43-57.

Hamsa, P. V. \& Chattoo, B. B. (1994). Cloning and growthregulated expression of the gene encoding the hepatitis $B$ virus middle surface antigen in Yarrowia lipolytica. Gene 143, 165-170.

Harding, T. M., Morano, K. A., Scott, S. V. \& Klionsky, D. J. (1995). Isolation and characterization of yeast mutants in the cytoplasm to vacuole targeting pathway. J Cell Biol 131, 591-602.

He, F., Beckerich, J. \& Gaillardin, C. (1992). A mutant 7SL RNA in Yarrowia lipolytica affecting the synthesis of a secreted protein. $J$ Biol Chem 267, 1932-1937.

von Heijne, G. (1986). A new method for predicting signal sequence cleavage sites. Nucleic Acids Res 14, 4683-4690.

Heslot, H., Nicaud, J. M., Fabre, E., Beckerich, J. M., Fournier, P. \& Gaillardin, C. (1989). Cloning of the alkaline extracellular protease gene of Yarrowia lipolytica and its use to express foreign genes. Proceedings of the 2nd SSM International Congress of Microbiology, Singapore, pp. 27-45.

Holst, B., Bruun, A. W., Kielland-Brandt, M. C. \& Winther, J. R. (1996). Competition between folding and glycosylation in the endoplasmic reticulum. EMBO J 15, 3538-3546.

Hunkapiller, M. W., Lujan, E., Ostrander, F. \& Hood, L. E. (1983). Isolation of microgram quantities of proteins from polyacrylamide gels for amino acid analysis. Methods Enzymol 91, 227-236.

Kreil, G. (1990). Processing of precursors by dipeptidylaminopeptidases: a case of molecular ticketing. Trends Biochem Sci 15, 23-26.
Kunkel, T. A., Roberts, J. D. \& Zakour, R. A. (1987). Rapid and efficient site-specific mutagenesis without phenotypic selection. Methods Enzymol 154, 367-382.

Lee, I. H. \& Ogrydziak, D. M. (1997). Yarrowia lipolytica SRP54 homolog and translocation of Kar2p. Yeast 13, 499-513.

Lopez, M. C., Nicaud, J. M., Skinner, H. B., Vergnolle, C., Kader, J. C., Bankaitis, V. A. \& Gaillardin, C. (1994). A phosphatidylinositol/phosphatidylcholine transfer protein is required for differentiation of the dimorphic yeast Yarrowia lipolytica from the yeast to mycelial form. $J$ Cell Biol 125, 113-127.

Matoba, S. \& Ogrydziak, D. M. (1989). A novel location for dipeptidyl aminopeptidase processing sites in the alkaline extracellular protease of Yarrowia lipolytica. J Biol Chem 264, $6037-6043$.

Matoba, S., Fukayama, J., Wing, R. A. \& Ogrydziak, D. M. (1988). Intracellular precursors and secretion of the alkaline extracellular protease of Yarrowia lipolytica. Mol Cell Biol 8, 4904-4916.

Nicaud, J. M., Fabre, E. \& Gaillardin, C. (1989). Expression of invertase activity in Yarrowia lipolytica and its use as a selective marker. Curr Genet 16, 253-260.

Ogrydziak, D. M. \& Mortimer, R. K. (1977). Genetics of extracellular protease production in Saccharomycopsis lipolytica. Genetics 87, 621-632.

Ogrydziak, D. M. \& Scharf, S. J. (1982). Alkaline extracellular protease produced by Saccharomycopsis lipolytica CX161-1B. $J$ Gen Microbiol 128, 1225-1234.

Sambrook, J., Fritsch, E. F. \& Maniatis, T. (1989). Molecular Cloning: a Laboratory Manual, 2nd edn. Cold Spring Harbor, NY: Cold Spring Harbor Laboratory.

Sherman, F., Fink, G. R. \& Hicks, J. B. (1986). Laboratory Course Manual for Methods in Yeast Genetics. Cold Spring Harbor, NY: Cold Spring Harbor Laboratory.

Tharaud, C., Ribet, A.-M., Costes, C. \& Gaillardin, C. (1992). Secretion of human blood coagulation factor XIIIa by the yeast Yarrowia lipolytica. Gene 121, 111-119.

Tobe, S., Takami, T., Ikeda, S. \& Mitsugi, K. (1976). Production and some enzymatic properties of alkaline protease of Candida lipolytica. Agric Biol Chem 40, 1087-1092.

Yaver, D. S., Matoba, S. \& Ogrydziak, D. M. (1992). A mutation in the signal recognition particle $7 \mathrm{~S}$ RNA of the yeast Yarrowia lipolytica preferentially affects synthesis of the alkaline extracellular protease: in vivo evidence for translational arrest. $J$ Cell Biol 116, 605-616.

Received 18 April 1997; revised 9 June 1997; accepted 16 June 1997. 